\title{
CUATRO REFLEXIONES PARA MEDITAR SOBRE EL QUEHACER EDUCATIVO
}

\author{
Marco Vargas Montero *
}

La educación no se limita a los procesos de enseñanza y de aprendizaje, pues también existen muchos temas, alrededor de ella, que, a menudo, nos hacen reflexionaar. Se presentan cuatro pequeños ensayos que desarrollan diferentes tópicos, aunque todos tienen que ver, de alguna manera, con la evaluación. Entre ellos estála (auto) valoración del profesormediante una serie de preguntas, la estimación del valor de la tecnología en la educación, la evaluación del docente en el desempeño de sus tareas y, por último, la apreciación de los sistemas universitarios público y privado. Todos los temas persiguen mejorar la calidad de diferentes as. pectos del ámbito educativo, en beneficio de todos, especialmente del que aprende.

Los talleres de educación de la sexualidad y la afectividad en los adolescentes como un medio para la prevención del abuso sexual (una propuesta metodológica y teórica).

\begin{abstract}
Education is not limited to the teaching and learning processes; there are many other related topics which often make us meditate. The following are four brief essays that explore different topics; yet, they all deal, in one way or another, with the evaluation process. Among them, the professor's self-assessment through a series of questions, estimating the importance of technology in education, evaluating the teacher while performing his tasks and finally, the appreciation of the public and private university systems. All the topics aim to improve the quality of the different aspects within the area of education, for the benefit of all, especially of those who learn.

The workshops on sex education and affectivity in adolescents as a means to prevent sexual abuse (a methodological and theoretical proposal).
\end{abstract}

* Profesor de Castellano y Literatura, Bachiller y Licenciado en Filología, Máster en Literatura, en Aprendizaje del español como lengua extianjera y en Docencia Universitaria. 
A veces me pongo a reflexionar en todos los adelantos que a través de los años de la existencia del ser humano se han hecho: descubrimientos científicos, inventos tecnológicos y muchos más en todos los campos, y pienso en que si se ha llegado tan lejos en ciencias, en artes, en letras y en tecnología deberíamos haber caminado lo suficiente en educación como para decir: "estamos satisfechos con lo que hemos conseguido en esta disciplina" y; sin embargo, creo que seguimos en pañales.

¿A qué se deberá esto? ¿Será que no nos importa cómo hagamos las cosas? ¿Será que no somos conscientes de lo importante de nuestro quehacer? ¿Será que nos equivocamos al escoger esta profesión? ¿Será que debe cambiar la forma de selección para mantener una propiedad en educación? ¿Será que creemos que cuando nos prepararon como profesores, lo hicieron tan bien que por eso no nos actualizamos? ¿Será que no nos ponemos a pensar que trabajamos con personas y no nos importa si las malformamos? ¿Será que no nos importa ser un mal modelo para nuestros estudiantes? ¿Será que nos gusta aplicar la ley del menor esfuerzo? ¿Será que nos importa solo la parte informativa y no la formativa? ¿Será que nos da lo mismo si la información que manejamos está obsoleta? ¿Será que nos hacemos resistentes al cambio? ¿Será que nos da lo mismo si planificamos o no, lo que 'enseñamos'? ¿Será que por tener un título consideramos que no tenemos nada más que hacer por la superación profesional? ¿Será que por tener propiedad creemos que somos inamovibles del puesto que desempeñamos? ¿Será que los estudiantes nos tienen que aguantar por el simple hecho de haber sido nombrados como profesores de un curso? ¿Será que el planeamiento de una clase se hace solamente cuando comenzamos a trabajar como profesores? ¿Será que la simpatía que poseemos puede sustituir la preparación académica? ¿Será que creemos que podemos engañar a los estudiantes por tener un título que ellos no tienen? ¿Será que no nos afecta si somos mal evaluados? ¿Será que los estudiantes son muy conformistas o despreocupados por la calidad de la enseñanza de un profesor? ¿Será que hay escasez de profesores? ¿Será que nos sentimos imprescindibles y que nadie puede hacer las cosas como las hacemos?

Creo que si respondemos sinceramente a estas preguntas y las interiorizamos, en algún momento se nos comenzaran a mover las fibras del orgullo, de la responsabilidad, de la honestidad y todas las fibras que se tengan que mover para empezar a realizar la labor docente mejor de lo que lo estamos haciendo. Para concluir, deseo parafrasear el mensaje que escriben al final de las cartas de recuerdo de pago, cuando dicen que: "Haga caso omiso de este aviso si ya se 
¿Será que los estudiantes nos tienen que aguantar por el simple hecho de haber sido nombrados como profesores de un curso?

¿Será que el planeamiento de una clase se hace solamente cuando comenzamos a trabajar como profesores? ¿Será que la simpatía que poseemos puede sustituir la preparación académica? ¿Será que podemos engañar a los estudiantes por tener un título que ellos no tienen?

o será la realidad? Sería interesante averiguarlo.

¿Por qué hablar de computadoras para las instituciones educativas?, pues porque la realidad ha cambiado: antes se hablaba de libros, cuadernos, pizarras; y como todo va cambiando, la educación también debe ajustarse a esos cambios. Cuando prevalecía (escrito en pasado, para creer que ya no) la utilización del paradigma conductista, era muy fácil para el maestro llegar a dar sus lecciones; con tiza, libros y, a veces, algún cartel, con lo que se sentía que 'cumplía' su labor. En este momento el maestro que haga eso es fuertemente criticado, pues así como ya no se concibe al aprendiz como un 'recipiente' que hay que llenar de conocimientos, tampoco se puede concebir al maestro como un 'recitador', que tiene que sacar todo lo que 'sabe'. En el caso del docente, principalmente, debe preocuparse por un cambio en su paradigma, lo que implica que modifique desde su planeamiento hasta la forma de evaluar, incluida, por supuesto, la metodología empleada.

Tradicionalmente, cuando se hablaba sobre ambiente educativo se pensaba en aquel dentro del cual se encontraba un grupo de alumnos, quienes 'recibían' los conocimientos que un maestro les 'ofrecía', pero en este momento ya no se considera de esa manera, ya que el ambiente educativo debe concebirse como aquel en donde todos aprenden y cada uno construye su conocimiento.

¿Cómo hacer para transformar un ambiente tradicional en uno moderno? La utilización de la tecnología en la educación es una manera de ir cambiando los conceptos de enseñanza y aprendizaje que se conocen, pues con ella se comienzan a romper los paradigmas educativos tradicionales, para abrir la puerta a nuevas formas de ver el mundo y cambiar los conceptos conocidos desde antes. Aunque las escuelas no puedan contar con todos los adelantos tecnológicos existentes, 
sí pueden contar (en ciertos casos) al menos con algunas computadoras; las que, bien utilizadas, pueden comenzar a promover el cambio en el sistema educativo.

Hasta hace poco tiempo, lo más 'moderno' en las escuelas era utilizar la televisión (V.H.S.) para apreciar alguna película y, con base en el contenido, realizar algunas actividades (en el mejor de los casos) preparadas previamente; en otras ocasiones, la película servía (y sirve todavía) para gastar tiempo, pues no se llevaba nada planeado y lo que pudo haberse explotado positivamente, se perdía después de apreciarlo.

Desde hace varios años se está implementando el uso de las computadoras, tanto en el aula como fuera de esta, pues se ha visto que tiene muchas ventajas en el desarrollo del proceso del aprendizaje-discente; entre otras el desarrollo de la creatividad, el despertar de la motivación por aprender, el desarrollo del aprendizaje cooperativo, la facilidad para construir el conocimiento y, por supuesto, la permanencia de este en cada uno de los individuos que participan en el proceso. Esto último, sin menospreciar lo anterior, es lo más valioso, ya que al fin y al cabo ¿qué es lo que persigue la educación si no es el enriquecer la mente de los aprendices y hacerlos mejores?

Pero, ¿es así de fácil el asunto: tener computadoras y poner a los alumnos al frente de ellas? No, claro que no. Es necesario dar una formación apropiada al profesor para que pueda desarrollar las habilidades cognitivas de los estudiantes, por supuesto que esto se dará si él mismo ha experimentado ese desarrollo, porque se dan casos en que un docente hace lo mismo que tradicionalmente hacía, pero ayudado por la computadora. Es imprescindible ese cambio de actitud en los maestros, pues si no se da esa transformación no tiene sentido realizar un gasto económico tan grande, como es el equipar un laboratorio de computación.

Con el equipo en la escuela se pueden llevar a cabo muchas actividades, como por ejemplo: solicitar un diario a cada estudiante, pedir que interactúen entre ellos, utilizar la computadora como una pizarra electrónica, establecer comunicación con otras instituciones, aprovechar las videoconferencias, trasladar información mediante internet, elaborar presentaciones y otras que, de acuerdo con la creatividad del docente, pueden multiplicarse.

$\mathrm{Y}$, ¿solo con un laboratorio en la institución puede trabajarse de esta manera? No necesariamente, en ciertos niveles, si no se tiene computadora en la clase se pueden organizar actividades y tareas para que los alumnos realicen fuera y se comuniquen con el profesor mediante la computadora de la casa y el correo electrónico (en el caso que tengan) o con las de los cafés internet, que ahora han proliferado tanto.

Es necesario pensar un poco más allá y no quedarse en el uso de la computadora en la clase y la interacción con el profesor, sino que en todas las instituciones se debería pensar en la computadora como una 'herramienta' obligatoria en toda biblioteca, pues si no se encuentra lo que se busca en un libro, se pueda 
consultar la biblioteca virtual, la que podría decirse, está al alcance de todos, en y desde cualquier lugar y, sobre todo, a cualquier hora.

Para concluir, se exponen dos ideas que deben quedar claras. En primer lugar, hay que recordar que en esta época, al profesor que desconozca y no maneje apropiadamente la tecnología con sus alumnos, puede llamársele analfabeta tecnológico y, en segundo lugar, que no se debe pensar, bajo ninguna circunstancia, que la tecnología sustituye al maestro; igual que otros instrumentos, esta es una ayuda para facilitar la labor y mejorar el ambiente educativo.

\section{Evaluación docente: una forma de mejorar la calidad}

"Me parece muy bien", "Le falta experiencia", "Eso me parece mejor que esto", "No me parece", "Creo que está bien, pero estaría mejor si...", "No me gusta", "Es excelente", "Tiene muchos errores para darle el visto bueno", "No sabe nada"; estas y muchas otras más son expresiones que se oyen a diario, en todos los niveles y en todos los ambientes. Esto se debe a que el ser humano es un evaluador por naturaleza: de todo aquello que lo rodea, de los actos y creaciones de los demás y, sobre todo, de las personas; muchas veces con un afán constructivo, pero otras, todo lo contrario: con una intención destructiva.

En los centros educativos, de una forma seria y con objetivos bien definidos se evalúan los proyectos y el desempeño de los funcionarios, con el fin de detectar los aspectos positivos que se necesita mantener y aquellos negativos que merecen la atención para mejorar, modificar y, en último caso, cambiar totalmente.

En el caso de los proyectos, por muy completos y bien hechos que estén, deben ser evaluados periódicamente, pues aunque hayan sido elaborados con cuidado y tomando en cuenta las mejores ideas, llega el momento en que necesitan una revisión para determinar los aciertos y los desaciertos que han tenido durante su período de funcionamiento y definir los cambios necesarios para que resulten de nuevo útiles, apropiados, atractivos y productivos. Este revisar, renovar y poner en práctica debe convertirse en una especie de banda sin fin, para que se siga repitiendo, mientras exista el proyecto. En las instituciones educativas existe una serie de proyectos que es necesario evaluar, con el fin de cambiar aquellos aspectos que lo ameritan para mejorar la calidad.

El desempeño de las personas también es evaluable, pues ellas también necesitan una realimentación, para modificar conductas, actitudes, conocimientos y habilidades, en pro de un mejoramiento personal, profesional e institucional. Es necesarísimo realizar la evaluación tanto de profesionales como de no profesionales, para mantener funcionando eficientemente el sistema. 
Es imperativo llevar a cabo la evaluación docente en las instituciones educativas, pues aunque existan otras áreas de desarrollo, como son la investigación, la extensión y la administración, la razón de ser de estas instituciones es la docencia y se debe mantener en un estado óptimo. Los estudiantes (quienes son verdaderos 'clientes') tienen derecho a recibir el mejor mato y una educación de calidad, porque no es productivo estar formando profesionales inseguros, manipulables y sin las 'armas' adecuadas para salir a competir a la 'selva' del mercado laboral. Ellos son la razón de ser de toda institución educativa y tienen el derecho de contar con los mejores docentes y con la mejor preparación personal y profesional.

Algunas razones que motivan la evaluación docente son las siguientes:

1. Todo docente ha sido contratado y es remunerado para realizar sus labores de la mejor manera; mediante la evaluación de sus alumnos se puede detectar cómo lo hace.

2. El profesor debe conocer cómo está llevando a cabo su labor, para fortalecer sus debilidades y mantener el buen desempeño en aquellos aspectos que así lo indican.

3. El superior inmediato debe conocer y dar cuenta a otras instancias acerca del cumplimiento de sus subalternos.

4. La evaluación pone alerta a todos los sujetos: al docente para que no se descuide en su quehacer; al superior para que se involucre en el proceso y observe cómo es el ejercicio de sus subalternos y a los estudiantes para que, objetivamente, 'defiendan' su derecho de contar con lo mejor.

5. Para buscar medidas correctivas, si fuera necesario, en el caso de evaluaciones bajas.

6. Para estimular el buen desempeño de los profesores.

7. Para mantener la excelencia, de la que tanto se habla en el ámbito universitario.

Para lograr lo anterior es necesario que todo el proceso esté bien planeado; que el instrumento de evaluación esté elaborado científicamente y, especialmente, que los resultados obtenidos sirvan para algo y no se queden 'ociosos' en un archivo. Es necesario que la evaluación sea obligatoria y vinculante entre los resultados y la estabilidad laboral; de otra manera, no tiene ningún sentido gastar el tiempo de los aplicadores y de los estudiantes, y el dinero de la institución, pues este proceso siempre resulta oneroso. Si el proceso se lleva a cabo seriamente, vale la pena cualquier gasto.

El hecho de que 'no pase nada', después de conocer los resultados, podría desembocar en una actitud de desidia general por parte de los más responsables 
y despertarse una sensación de desconfianza en el sector estudiantil; lo que conduciría a un caos dentro de la institución.

Es imprescindible que se realice la evaluación docente en las instituciones educativas para mantener la calidad que exige la sociedad y, sobre todo, para ofrecerle a esta los mejores profesionales del futuro.

\section{Eterna polémica nacional}

La mayor parte de las personas tienen por costumbre establecer comparaciones de todas aquellas cosas que la rodean, sean estas personas u objetos; así se dan parangones entre los hijos, los docentes, los vecinos, los carros, las casas, los comportamientos y todo lo demás. Diría, como reza el dicho popular: "Toda comparación es odiosa"; sin embargo, uno mismo cae, en algún momento, en esas odiosas comparaciones.

En Costa Rica se ha puesto muy de moda comparar los sistemas de educación pública y privada, especialmente el nivel de educación superior y se establecen verdaderos debates, pues algunos defienden la universidad pública y atacan la privada; otros, todo lo contrario. ¿Quién tiene la razón en esta discusión? Especulemos un poco, al respecto, con una posición objetiva.

En Costa Rica existen cuatro universidades estatales: la Universidad de Costa Rica, fundada en 1940; el Instituto Tecnológico de Costa Rica, creado en 1971; la Universidad Nacional, fundada en 1973 y la Universidad Estatal a Distancia, de 1977. La primera universidad privada (UACA) fue creada en 1977 por un prestigioso grupo de universitarios independientes, lo que motivó gran revuelo, motivó la crítica y a partir de ahí se estableció un debate nacional que no ha parado y del cual nadie ha escapado; se quiera o no, todos los docentes nos sentimos involucrados en esta discusión. La basura de ese debate cae, por supuesto, sobre la universidad privada, por creer que lo estatal, lo público, lo barato, lo establecido, lo tradicional, ... es mejor; pero de vez en cuando la universidad pública se siente salpicada por esa basura. Toda esta polémica gira alrededor de la calidad de las instituciones universitarias.

El número de las universidades privadas ha ido creciendo, al principio tímidamente; pero desde hace unos años, han proliferado en forma desmesurada y en este momento existen unas 50 universidades privadas en todo el país ... y sigue aumentando el número.

Los que atacan a las instituciones privadas dicen que son universidades de garaje, pero algunas tienen una infraestructura tan completa que cualquier universidad estatal se la desearía. Alegan que lo único que les interesa es atraer estudiantes para recibir más ganancias económicas; podría ser, pero a nadie se 
obliga a matricularse en ellas, quien estudia ahí lo hace por su voluntad. Aseguran, también, que los estudios que ofrecen son de menor calidad que el ofrecido por las públicas y que los profesores no están bien preparados; pero ... ¿no son los mismos profesores de las públicas?, ¿por qué van a ser peores? Hay buenos profesores tanto en el sistema privado como en el público.

Los defensores de las universidades públicas insisten en que estas son mejores porque a los alurnnos se les ofrece una mejor formación humanística, claro que hay que ponerse a pensar que esa formación alarga la carrera en uno o dos años; en las privadas al no tener que cursar estos requisitos se gradúan más rápido y comienzan a percibir un salario más pronto. Aseguran que los estudiantes de las públicas están mejor preparados porque han ganado un examen de admisión o han sido admitidos mediante un proceso de selección estricto; pero tampoco es un argumento de peso, pues los que entran en las privadas estarían igualmente preparados para enfrentarse a un proceso de admisión, porque han ganado las pruebas nacionales de Bachillerato.

Los atacantes de las privadas afirman que los que estudian en ellas son los 'hijos de papi' y que no tienen buenas costumbres; sin embargo, esa afirmación no es del todo cierta, ya que muchos trabajadores estudian en este sistema y a ellos sí les cuesta pagar los estudios, no se los paga 'papi' y, además, en todos los ámbitos se encuentran personas 'buenas' y 'malas'.

Podría seguir escribiendo razones para desacreditar a unas o acreditar a las otras, pero creo que para cualquier argumento en contra existe otro a favor y viceversa, por io que sería de nunca acabar. De todas formas he aprendido a no meter las manos al fuego por defender cosas que yo no puedo controlar, porque se me pueden quemar. Creo que en ambos sistemas hay de todo: ventajas y desventajas, economía y derroche, responsabilidad e incompetencia, mística e interés económico, ... y, nuevamente, tengo que decir que se puede seguir enumerando pares de características, pero tendría que gastar muchas líneas y a lo peor no llegaría a una conclusión satisfactoria.

En lo que sí han comenzado a diferenciarse los dos sistemas, desde hace varios años, es en el proceso de acreditación, que se está dando tanto a escala nacional como intemacional, pues las cuatro universidades públicas están inscritas en el SINAES (Sistema Nacional de Acreditación de la Educación Superior) y del gran total de instituciones privadas solamente siete de ellas (de las más grandes) se han inscrito. ¿Por qué será que no lo han hecho las otras?

Para concluir, he de decir que hace muchos años, cuando había menos estudiantes bastaba con pocas universidades públicas, pero en este momento hay que preguntarse: ¿dónde podrían seguir los estudios universitarios, los miles de estudiantes que se gradúan año con año de secundaria? El que encuentre la solución a 
este problema sería digno merecedor de un premio, porque la situación es difícil y cada día se acentúa más el problema. ¡Ojalá llegara un día cuando pudiéramos hablar de la buena educación superior, sin distinguir si es pública o privada!

Yo tengo mis firmes creencias acerca de los dos sistemas, ¿y usted?

El juicio externado en estas reflexiones podría ser que no coincida con la opinión de los lectores; pero se debe tener presente que en un ensayo se expresa un punto de vista muy personal y, además, que no se pretende agotar el tema, sino que se persigue dejar una enseñanza, mover a la discusión o formar criterio sobre el tema tratado.

¿Qué piensa, usted, como educador(a) acerca de las ideas expuestas? 